\title{
The syrian national kidney foundation: Response for the need of kidney patients during the crisis
}

\section{INTRODUCTION}

Before the development of acute hemodialysis, mortality rate of acute renal failure (ARF) approached $100 \%$ in World War II. ${ }^{[1]}$ Most of these kidney injuries were caused by crush injuries as described by Bywaters and Beall. The use of hemodialysis was first described in military services during the Korean War in 1950 for renal military casualties; this has led to a decline in mortality rate from around $90 \%$ to $53 \% \cdot{ }^{[2,3]}$ Early intervention could prevent the occurrence of ARF, at least in military casualties. ${ }^{[4]}$ During disasters and War situations, the delivery of healthcare services including hemodialysis can be interrupted due to destruction of medical facilities and infrastructure, lack of healthcare personnel, shortage of equipment and supplies, and interruption of electricity and water. The conduction of hemodialysis can also be interrupted by technical or electrical issues such as electrical power blackouts. ${ }^{[5]}$

The uprising demand for political change in Syria that started from March 2011 has marked the beginning of an ongoing Syrian political and humanitarian crisis. This conflict has led to a significant decline of living standards, loss of healthcare facilities, flight of medical personnel, severe shortage of medicines, lack of essential supplies and an increased risk of infectious diseases in the affected areas. According to the World Health Organization (WHO), the crisis context has compromised the provision of primary and secondary healthcare, the referral of injured patients, treatment of chronic diseases, disruption of maternal and child health services, vaccination and nutritional programs and that of communicable disease control. ${ }^{[6]}$

Renal care and hemodialysis patients were not the exception. The complexity and the requirements of the hemodialysis procedure magnified the problem in delivering quality,

\begin{tabular}{|l|l|}
\hline \multicolumn{2}{|c|}{ Access this article online } \\
\hline Quick Response Code: & Website: \\
\hline & www.avicennajmed.com \\
\hline & \\
\hline
\end{tabular}

effective and safe services. All or some components of renal care were inadequate or completely absent due to security issues, unavailability of supplies, interruption of water and electricity, absence of maintenance and support, and absence of equipment and essential materials. Accurate figures are lacking due to scarce and limited data from the area and most of the information is based on direct observation and rare reports.

Many medical relief efforts were organized by non-governmental organizations (NGOs), including the Syrian American Medical Society (SAMS), to provide medical and psychological support to the internally displaced people, people in conflict-affected areas and Syrian refugees in surrounding countries. ${ }^{[7]}$ Among the different medical missions of Syria, SAMS was the mission of the Syrian American Nephrologists to refugee camps and Northern Syria with the objective of providing a preliminary assessment of the care delivery status of renal patients. Their direct observation revealed that the care of dialysis patients was severely compromised due to lack of access to dialysis units, electricity outage, lack of medications and equipment, destruction of healthcare facilities and shortage of medical care providers. The majority of dialysis facilities had no supervising nephrologists; some of the provinces lacked the existence of any nephrologist. The majority of ARF was caused by crush injuries and rhabdomyolysis (54\%) followed by gunshot injury (35\%) per the observation of one of SAMS physicians who visited northern Syria. Mortality figures were not available due to destruction of information system and lack of any data collection, many deaths occurred in fields due to massive hemorrhage, crush injuries and the lack of appropriate resuscitation. Victims who survived the initial shock have developed ARF-acute kidney injury (AKI) and most of them died because of complications of ARF-AKI since renal replacement therapy (RRT) was not available. Some renal transplant patients had rejection and ended up on dialysis because of inability to find or afford anti-rejection medications.

\section{Renal disease and renal care in Syria}

There is not enough data in the medical literature regarding the status of renal care in Syria before or after the crisis. We do not know the true incidence of end stage renal disease (ESRD) due to lack of national registry. WHO 
website did not publish any data or statistics in regard to renal care in Syria. A Medline search revealed one article published in 2009 entitled "Epidemiology of Hemodialysis Patients in Aleppo City" where it was reported that a total of 550 dialysis patients existed in the city of Aleppo. ${ }^{[8]}$ In May 2005, the annual report of renal replacement therapy (RRT) in Syria, which was issued by department of the ministry of health, revealed that there were 2750 new patients on hemodialysis (HD) and 111 patients on continuous ambulatory peritoneal dialysis (CAPD). ${ }^{[9]}$ In an estimate, from a physician who used to work at the Syrian Department of Health and now collaborates with SNKF, the total number of dialysis patients before the war started was about 7000 .

Renal and dialysis patients would have been the most vulnerable people among all chronic diseases during the conflict and their care would have been affected majorly due to their fragile status, co-morbidities and the complexity of offered treatments.

There are numerous anecdotal reports by SNKF members indicating the major impact of the crisis on the functionality of hemodialysis units and dialysis machines. For example, in one observation of a hemodialysis center in Aleppo; the electricity was available through a diesel-run generator with 3-4 shifts per day for six days with Friday being only for emergencies and is the only day-off for the technicians. There was one machine for hepatitis B isolation; and the center tried to make a "hepatitis B shift" as much as possible. Fistulas and temporary subclavian catheters were the main vascular access. There was no interventional care for maturation or maintenance of the fistulas and the catheters are kept for very long duration (sometimes 2-3 years). No tunneled catheters were used due to cost and no grafts due to cost and lack of surgical experience with implantation. The concepts of venous vascular balloons, stents, or decloting did not exist. Cannulation of those fistulas was sometimes challenging but the technician has adapted very well to the procedure. Temporary subclavian catheters are commonly used. The rate of line infections was probably underappreciated and usually treated with empiric antibiotics without replacement or exchange. The lines were not placed under ultrasonographic guidance. The standard of care regarding the frequency of dialysis was one per week and sometimes two but very rarely to have dialysis schedule of three times a week except if the patients were financially secure. Many patients did not understand the concept of chronic hemodialysis and the ongoing need for that. This was especially true for new starts. Dialysis baths were usually $2 \mathrm{~K}(2 \mathrm{meq} / \mathrm{L})$ premade without any other option for other concentrations for the acid. The base was made from powder and was quite cheap and available. The facility also accepted all non-critical patients including primary care both adults and pediatrics. Critically ill patients were transported to bigger centers after stabilization. The facility was shared with ophthalmology, wound and burn care. It had minimal security and no internet connection. The SNKF team concluded that lack of physician support is a major cause of less than the optimal delivered renal care. However, the current staff was doing a great job compared to the resources they have, whether that be in- personnel, knowledge or equipment. Physician support and supervision could be re-established if the facility gets a satellite internet connection.

\section{Syrian national kidney foundation}

The Syrian National Kidney Foundation (SNKF) idea was established by two Syrian American nephrologists in San Diego during the American Society of Nephrology (ASN) Renal Week in November 2012.

Initially, the mission was to deliver care to the Syrian renal patients in need. Overtime, it was refined to:

- Facilitate, support, and deliver care to the renal and dialysis patients in Syria through collaboration with different organizations.

- Support staff and providers with objective of prioritizing needs and maximizing benefits despite limited resources.

- Standardize the nephrology practice and improve outcome of renal patients in Syria.

The vision is to become the foundation of kidney diseases in Syria for all nephrologists.

The objective is to have a structured approach towards improving delivery and quality of care for dialysis, renal transplant and AKI patients. The group consists of nephrologists, biomedical engineers, dialysis technicians, administrators, and Syrian internists involved in the care of kidney patients. The group meets weekly via Skype. The core functions of the group are shown in Table 1.

The first official meeting was in February 2013 during the national conference of the SAMS held in Tampa, Florida. A detailed report from a returned physician from Syria confirmed the complete lack of nephrologists in some areas and the shutdown of many hemodialysis units in other areas. In neighboring countries such as Lebanon and Turkey, patients were receiving hemodialysis with different standards and supported form different charity organizations. There were at least 30 hemodialysis patients in northern Lebanon with lack of essential medicines including erythropoiesis-stimulating agents. The plan was to raise 
funds from the foundation members and through different channels for a purchase of dialysis machine. They also worked on conducting the first in-service for local physicians, nurses and technicians by maintaining communication and follow-up through regular telecommunication and Skype.

A follow-up meeting was held in Atlanta on March 24, 2013 where discussion took place on what has been accomplished and on the need of the establishment of a complete dialysis unit with a stable budget to operate and maintain. More roles that are active among the nephrologists took place to increase awareness about the foundation. Personal contacts and emails were used and channels in social media such as Facebook and YouTube were opened. ${ }^{[10,11]}$ This led to an increased recruitment of nephrologists from around the world into the foundation, with a total number reaching 35 , and increased missions to Syria and refugee camps. A weekly renal transplant clinic was started with the support of internist staff providing support via internet by a US transplant nephrologist. More financial support has led to the establishment of the dialysis unit in Idlib, operate it and maintain it with supplies, medications along with a continued education and support from nephrologists. The facility is located in rural Idlib, using 2 hemodialysis machines and supports 18 ESRD patients.

Novel and cost effective ideas helped providing new solutions for RRT in the intensive care unit (ICU) for AKIpatients. An Improvized setup for continuous veno-venous hemofiltration $(\mathrm{CVVH})$ was developed, standardized, tested and successfully implemented. It was simplified to be used in harsh conditions, like using a car battery as an electrical source. [Figure 1] Staff training was simple, hands on and YouTube videos were produced, ${ }^{[11]}$ as well as protocols were developed taking into consideration the available local resources. Remote real time Skype support was available as well. By the time this paper is written, 6 patients were saved using this method. The SNKF physicians are in the process of writing a case series describing this accomplishment and will be submitted to a peer review journal. A summary of SNKF accomplishments so far is shown in Table 2.

While trying to provide safe treatment, the war may require that the nephrologist set aside his or her standard approaches and guidelines and seek innovative ways in order to maximize outcomes for the greatest number of individuals using limited recourses. ${ }^{[12]}$

SNKF progressed from a group of doctors working individually to a legitimate and organized body working on a comprehensive project to improve health care in general and dialysis patients particularly in Syria at time of war

\begin{tabular}{|c|c|}
\hline Core function & Current activities \\
\hline Assessment & $\begin{array}{l}\text { Survey of Syrian dialysis facilities and their providers } \\
\text { Survey of dialysis and kidney transplant patients } \\
\text { Evaluation of funding and support resources } \\
\text { Evaluation of dialysis projects proposed by Syrian } \\
\text { institutions }\end{array}$ \\
\hline Assurance & $\begin{array}{l}\text { Online and telemedicine consultations to Syrian } \\
\text { providers } \\
\text { Visits to Syria by Syrian nephrologists and biomedical } \\
\text { engineers } \\
\text { Creation of technologies suitable for the } \\
\text { limited-resources environment } \\
\text { Communications with potential funding resources } \\
\text { regarding donations and sponsoring particular projects }\end{array}$ \\
\hline Education & $\begin{array}{l}\text { Publications of manuals and instructional videos on } \\
\text { various aspects of renal care } \\
\text { Participation in face-to-face educational courses to } \\
\text { Syrian providers } \\
\text { Educate potential donors about kidney disease and its } \\
\text { treatment } \\
\text { Sharing acquired knowledge by publishing the group's } \\
\text { findings in medical journals }\end{array}$ \\
\hline Policy & $\begin{array}{l}\text { Authoring management protocols that take into } \\
\text { consideration the limited resources }\end{array}$ \\
\hline
\end{tabular}

\section{Table 2: Some SNKF accomplishments}

Weekly nephrology and renal transplant clinics via internet by US nephrologists

Establishment of the dialysis unit in Idlib Hospital

Online support for acute dialysis, CRRT and ICU in multiple hospitals in Aleppo

Shipment of 30 dialysis machines from USA to Syria, and training staff to use the machines

Reaching out to different relief organizations and securing grants

Research, registry, and publication regarding dialysis services in Syria Issuing and posting dialysis treatment protocols and management of dialysis related illness

Training courses in dialysis and renal care for physicians and technicians

ICU: Intensive care unit, SNKF: Syrian national kidney foundation, CRRT: Continuous renal replacement therapy

and in the future peace. The work of SNKF will add to our current knowledge about renal care in disaster situations. The knowledge currently stems mostly from lessons learned while taking care of crush syndrome patients and during major earthquakes. ${ }^{[13]}$

The foundation is still in the establishment phase and growing steadily as planned, but much more is needed to accomplish its mission and the foundation members agreed to proceed based on the following approach:

- Strengthen the organizational structure of the foundation and establish key offices including a quality assurance, human resource and operation coordinator offices

- Build a sustainable capacity of the foundation through direct interaction and collaboration with different regional and international organizations

- Expand aid responses by developing a strategy that 


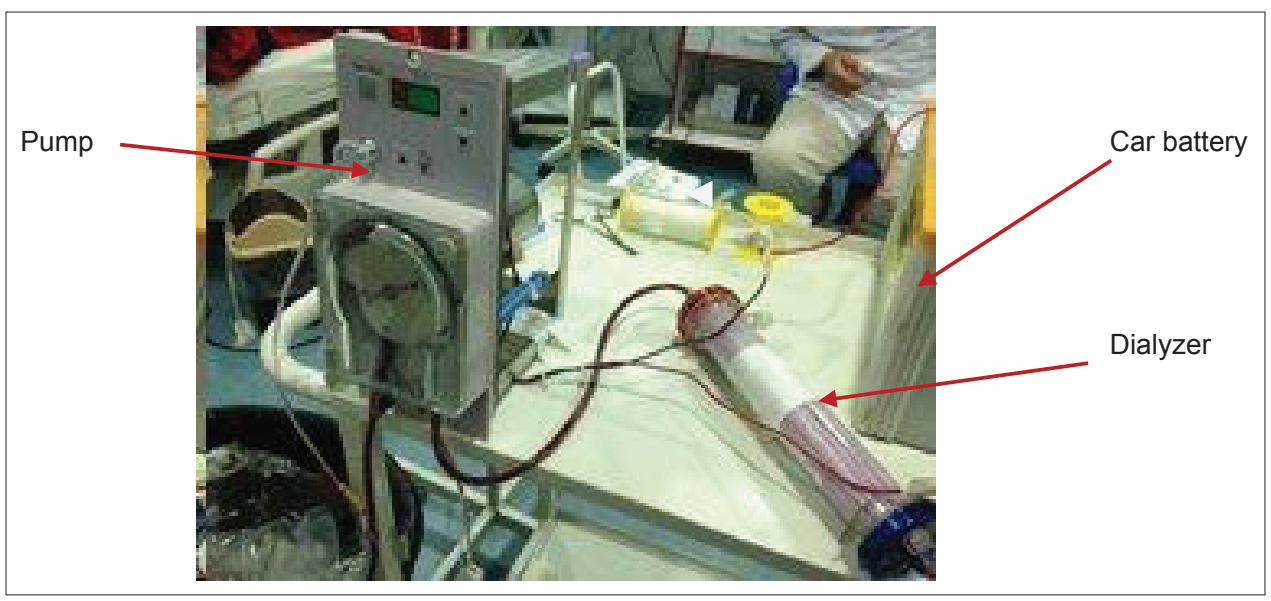

Figure 1: CVVH using a stand-alone blood pump for ARF in field hospitals in Syria. A car battery used as source of electricity

addresses renal care in other areas such as Turkey and Lebanon and develop an emergency preparedness plan for AKI and CKD patients in these areas

- Increase the awareness of public as well as physicians to the foundation through different media promotion and advertisement programs

- Establish an operation budget, secure sources for income, establish mechanisms to ensure accountability and enhance donations and volunteerism.

\section{CONCLUSION}

Teamwork between Syrian American Nephrologists, other nephrologists from around the world, technicians, engineers, and Syrian physicians has translated into a reasonably safe and effective treatment for Syrian renal patients in this dire situation. The SNKF will continue to progress, to achieve its objectives in developing an emergency preparedness plan for AKI and CKD patients and improving outcome of those patients. The structure, approach and experience of the SNKF could be a guide to other Syrian and non-Syrian provider groups involved in healthcare delivery in disastrous situations. More studies on the care of renal casualties during crisis in resource-limited counties are desperately needed.

Akram Al-Makki, A Oussama Rifai ${ }^{1}$, Lina Murad ${ }^{2}$, Abdul R Zanabli³, Anas Kayal4, Khaldoun Soudan5, Mazen Kherallah' ${ }^{6}$,Fahd Alsaghir7, Mohamed Sekkarie ${ }^{8}$ Indiana University Health Arnett, Lafayette, Indiana, ${ }^{1}$ Hypertension Kidney and Dialysis Specialists, Panama City, Florida, ${ }^{2}$ Metropolitan Access Center, Colmar Manor, Maryland, ${ }^{3}$ Charleston Nephrology, Hypertension, and Transplant, S Charleston, West Virginia, ${ }^{4}$ Ruston Nephrology Associates, Ruston, Louisiana,

${ }^{5}$ Freedman Clinic of Internal Medicine, Alexandria, Louisiana, ${ }^{6}$ University of North Dakota, Fargo, North Dakota, ${ }^{7}$ Michigan Kidney Consultants in Pontiac, MI, ${ }^{8}$ Nephrology and Hypertension Associates, Bluefield, West Virginia, USA E-mail: almakkia@iuhealth.org

\section{REFERENCES}

1. Bywaters EG, Beall D. Crush injuries with impairment of renal function. Br Med J 1941;1:427-32.

2. Teschan, PE, Post RS, Smith LH Jr, Abernathy RS, Davis JH, Gray DM, et al. Post-traumatic renal insufficiency in military casualties. I. Clinical characteristics. Am J Med 1955;18:172-86.

3. Smith LH Jr, Post RS, Teschan PE, Abernathy RS, Davis JH, Gray DM, et al. Post-traumatic renal insufficiency in military casualties. II. Management, use of an artificial kidney, prognosis. Am J Med 1955;18:187-98.

4. Schrier RW, Wang W, Poole B, Mitra A. Acute renal failure: Definitions, diagnosis, pathogenesis, and therapy. J Clin Invest 2004;114:5-14.

5. National Kidney Foundation. Planning for Emergencies. A Guide for People with Chronic Kidney Disease 2010. [Homepage on the Internet]. Available from: http://www.kidney.org/atoz/pdf/disaster_prepardness. pdf [Last cited on 2013 Nov 11].

6. WHO. Regional situation report. 2013. [Homepage on the Internet]. Available from: http:/www.emro.who.int/images/ stories/syria/documents/Syria_crisis_SitRep_WHO_18_01_Oct.pdf [Last cited on 2013 Nov 2].

7. Alghothani N, Alghothani Y, Atassi B. Evaluation of a short-term medical mission to Syrian refugee camps in Turkey. Avicenna J Med 2012;2:84-8.

8. Moukeh G, Yacoub R, Fahdi F, Rastam S, Albitar S. Epidemiology of hemodialysis patients in Aleppo city. Saudi J Kidney Dis Transpl 2009;20:140-6.

9. Saeed B, Derani R, Hajibrahim M, Roumani J, Al-Shaer MB, Saeed R, et al. Organ failure in Syria: Initiating a national deceased donation program. Saudi J Kidney Dis Transpl 2007;18:270-6.

10. Syrian National Kidney Foundation Project. 2013. [Homepage on the internet]. Available from: https:/www.facebook.com/pages/ Syrian-National-Kidney-Foundation-Project/574054545978685 [Last cited on 2013 Nov 11].

11. Syria Nephrology. 2013. [homepage on the internet]. Available from: http://www.youtube.com/channel/UCohWL6GhlyIW4w6PxOUWPhA [Last cited on 2013 Nov 11].

12. Yuan CM, Perkins RM. Renal replacement therapy in austere environments. Int J Nephrol 2011;2011:748053.

13. Raman G, Perkins RM, Jaar BG. Acute kidney injury and ESRD management in austere environments. Adv Chronic Kidney Dis 2012;19:149-57.

Cite this article as: Al-Makki A, Rifai AO, Murad L, Zanabli AR, Kayal A, Soudan $\mathrm{K}$, et al. The syrian national kidney foundation: Response for the need of kidney patients during the crisis. Avicenna J Med 2014;4:54-7.

Source of Support: Nil, Conflict of Interest: None declared. 